\title{
Growth factors in central nervous system development and tumorigenesis
}

\section{Barbara E. Lazio, M.D., and Lawrence S. Chin, M.D. \\ Department of Neurosurgery, University of Maryland School of Medicine, Baltimore, Maryland}

Growth factors play an important role in the development of the normal central nervous system as well as in the genesis of central nervous system tumors. Some of the more important growth factors and growth factor receptors, as they pertain to neurooncology, are reviewed in this article.

Key Words * growth factor * tumorigenesis * cell biology

A series of specific extracellular signals (neurotransmitters, hormones, and growth factors) are necessary for any cell to divide and differentiate. Most of these molecules (ligands) are polypeptides that bind selectively to receptors on the cell membrane, activating an intracellular cascade of other polypeptides (second messengers), and transmitting a message to the cell's nucleus. Many tumor cells have mutations that allow uninhibited production of a growth factor, resulting in uninhibited growth.[1]

A family of growth factor receptors, the tyrosine kinases (TKs), has been found to be important in normal and abnormal central nervous system (CNS) cell growth;[20,32,35] specifically, these receptors are epidermal growth factor (EGF), platelet-derived growth factor (PDGF), insulin-like growth factor (IGF), fibroblast growth factor (FGF), vascular endothelial growth factor (VEGF), nerve growth factor (NGF) and related ligands, brain-derived growth factor (BDGF) and neurotrophin-3 (NT-3).

The TK growth factor receptors are transmembrane polypeptides with a ligand-binding domain on the extracellular segment, a hydrophobic transmembrane segment, and an intracellular TK segment. The TK segment is the catalytic domain that acts by phosphorylating first the receptor and then any of several intracellular substrates. This activates the mitogenic second messenger systems within the cell and, with continued growth factor stimulation, leads to cell division. In a neoplastic environment, amplified growth factor expression can provide enough stimulus to the TK receptors (TKRs) to cause cells in the $\mathrm{G}_{0}$ phase of mitosis to progress through the cell cycle.[1]

The biological effects that the TKRs elicit can vary based on the growth factor, cell, and environment. Although most receptors stimulate growth, they may stimulate arrest of growth and differentiation given the appropriate cell and environment. For example, when rat pheochromocytoma cells are stimulated by NGF, which activates the trk family of neurotrophin receptors, the cells differentiate into cells resembling sympathetic neurons.[5] Conversely, when the same NGF stimulus is given to fibroblast 
cells, it induces rapid proliferation.

\section{GROWTH FACTORS AND TKRS IN CNS DEVELOPMENT}

Growth factor receptor families that have relevance to the CNS include the EGF receptors (c-erbB, c-erbB-2 [neu], and tyro-2), the neurotrophin receptors (NGFR [trk], BDNFR [trkB], and NT-3R [trkC]), the VEGF receptors (flt, Tiel,2, and flk-1), the IGF receptors (insulin receptor, IGF-1R, and met), the FGF receptors (FGFR1 [flg], FGFR2 [bek], FGFR3, and FGFR4), and the PDGF receptors (PDGFR-A, PDGFR-B, CSF-1R [c-fms], and c-kit).[35] Many of these receptors were originally identified as oncogenes. For example, the trk family of receptors was originally identified as an oncogene associated with DNA from human colon carcinoma.

The neurotrophin receptors NGFR, trk, trkB, and trkC have been shown to be expressed in higher concentrations in mature and developing neural tissues[20] and play an important role in the survival and differentiation of neural crest and central cholinergic neurons.[35] Klein, et al.,[16] illustrated the significance of the trkB gene in the neurological development of mice by rendering the trkB gene nonfunctional in embryonic stem cells and then inserting the stem cells into blastocysts. The blastocysts were inserted into the uteri of pregnant mice and were carried to term. The offspring, heterozygous for the mutation, were crossbred. The second generation offspring that were homozygous for the trkB mutation showed fewer than normal neurons in several areas of the central and peripheral nervous system (spinal cord, facial motor nuclei, and trigeminal and dorsal root ganglia).

Fibroblast growth factor and FGF TKRs have also been shown to be influential in nervous system development. Peters, et al.,[30] showed that in embryonic mice, neurons and glia are derived from the same precursor cells in the germinal epithelium. These cells express both FGFR1 and FGFR3 in the embryonic germinal epithelium, whereas in adult mice, FGFR1 is found predominantly in the hippocampus, cerebellar granule cells, and brainstem nuclei, and FGFR3 is found predominantly in glial cells. For this reason, exposure of the FGFR1 and FGFR3 in the germinal epithelium to FGF is thought to influence the differentiation and proliferation of these cells.

Other growth factors and receptors important in CNS development include, EGF, IGF, PDGF, and the recently identified glial growth factors. The glial growth factors are expressed in the CNS during embryonic development of Schwann cells and bind to the c-erbB2 receptor of the EGF family.[24] Epidermal growth factor has been shown to influence differentiation of precursor cells in the striatum and retina.[34] Platelet-derived growth factor is influential in the development of oligodendroglia. Insulin-like growth factor-1 and IGF-2, interacting with the IGF-1R are important in the growth and development of neuroglial cells.[35]

When the growth factors act sequentially, the responses are different than if cells are exposed to each factor individually. For instance, NGF can stimulate proliferation in embryonic neuroblast cell in the striatum, but only if the cells are primed with basic (b)FGF.[34]

\section{GROWTH FACTORS AND TKRS IN CNS NEOPLASIA}

Neoplasia occur when the usual relationship between cell proliferation, survival, differentiation, and apoptosis are disrupted. Tumorigenesis may occur when a transforming event uncouples the normal progression from proliferation to differentiation. The normal growth controls may be interrupted by a variety of mutations, causing overproduction of growth factors or of growth factor receptors, and keeping cells in a persistent state of proliferation.[7] In fact, two growth factors, PDGF and FGF, were originally 
identified not for their roles in normal development, but for their roles in tumorigenesis.[7] The growth factor receptors that are implicated in tumor formation in the CNS are similar to those important in normal development: EGF, PDGF, bFGF, and VEGF.

One EGF receptor, encoded by the c-erbB-2 (neu) oncogene, has been implicated in rat neuroblastomas and in human glioblastomas.[22] Amplification of the EGFR messenger RNA up to 10 times the normal expression has been found in a subset of glioblastomas. Often the receptors encoded by these amplified genes are abnormal, with part or all of the ligand-binding domain missing and/or mutations that enable the TK segment to function without a ligand at all.[22] In addition, overexpression of the growth factor ligands (EGF and transforming growth factor- $\beta$ ) has been associated with amplified expression of the receptors, implicating a possible feedback loop to the expression of EGFR and its ligands.[21]

Platelet-derived growth factor and its receptors have been demonstrated in astrocytic tumors: PDGF-A has been implicated in tumor proliferation, whereas PDGF-B has been more closely associated with angiogenesis in tumors.[21,35] Both growth factors are found in tumor cells that coexpress their specific receptors (PDGFR-A and PDGFR-B, respectively). The mechanism by which these astrocytic tumor cells overexpress PDGF/PDGFR seems to be related more to an autocrine growth stimulatory feedback loop than to gene amplification.[21] There is evidence that PDGF antagonists can arrest or slow the growth of astrocytic tumors in vitro.[6,17]

Vascular endothelial growth factor, a vascular permeability factor, may be overexpressed in human glioblastoma cells. The VEGF receptor flt is a TKR and has also been expressed in the vascular endothelium of glioblastomas, but not in the vasculature of the normal brain.[21] Inhibition of the VEGF receptor flk has been shown to inhibit growth of rat brain tumors in vivo. Acidic FGF and bFGF are found in higher concentrations in malignant astrocytomas than in normal brain and are also thought to play a role in angiogenesis in high-grade gliomas.[28] In addition, vascular permeability factors play a role in cerebral edema associated with meningiomas. A strong correlation exists between the extent of peritumoral edema found on imaging studies and VEGF immunoreactivity in meningioma cells.

Insulin and the IGFs are thought to play a role in the growth of human meningioma, low-grade glioma, and glioblastoma.[13] Insulin growth factor-1 and IGF-2 have been shown to bind to glioma cells and increase their proliferation.[13] The mitogenic effects of the IGFs are mediated through TK activity of the IGF-1R. Inhibition of this receptor by antibody or antisense can inhibit growth of brain tumor cells in culture.

\section{GROWTH FACTOR SIGNAL TRANSDUCTION}

Typically both neoplastic and nonneoplastic cells are dependent on trophic or growth factors to help with proliferation. These factors may be derived from local tissues (paracrine stimulation) or may be secreted by the cells themselves (autocrine stimulation). Loss of these growth factors can trigger cell death, as seen in the developing nervous system when large numbers of neuronal cell precursors undergo apoptosis due to limited amounts of neurotrophic factors.[5] Inhibiting the growth factors that activate glioma cells is therefore a logical approach to blocking their proliferation; however, the large number of different growth factors that can stimulate glioma cells makes this an impractical solution. A different approach is to track the growth factor signal inside the cell and dissect the pathways it uses to induce cell division. Once understood, these pathways may be inhibited at key points to block the signal from growth factors activating the cell. 
Insulin-like growth factor-1, PDGF, and EGF are known to support glioma cell growth in culture.[11,25,36] Furthermore, glioma cells express these growth factors in vivo.[14] Growth factors interact with membrane-bound receptors that typically show TK activity. After binding the appropriate growth factor, TKRs dimerize in the cell membrane and undergo autophosphorylation, which activates the TK domain.[33] Following receptor activation, a number of signaling pathways are available (Fig. 1). The three most significant pathways are controlled by phosphatidylinositol-3 (PI-3) kinase, which phosphorylates membrane proteins and is inhibited by wortmannin; phospholipase-C, which activates protein kinase $\mathrm{C}$ (PKC); and Ras, which appears to be the dominant pathway used for growth factor signaling.[9] A complicating feature is that all three pathways seem to have the ability to "cross-talk" and influence each other.[15]

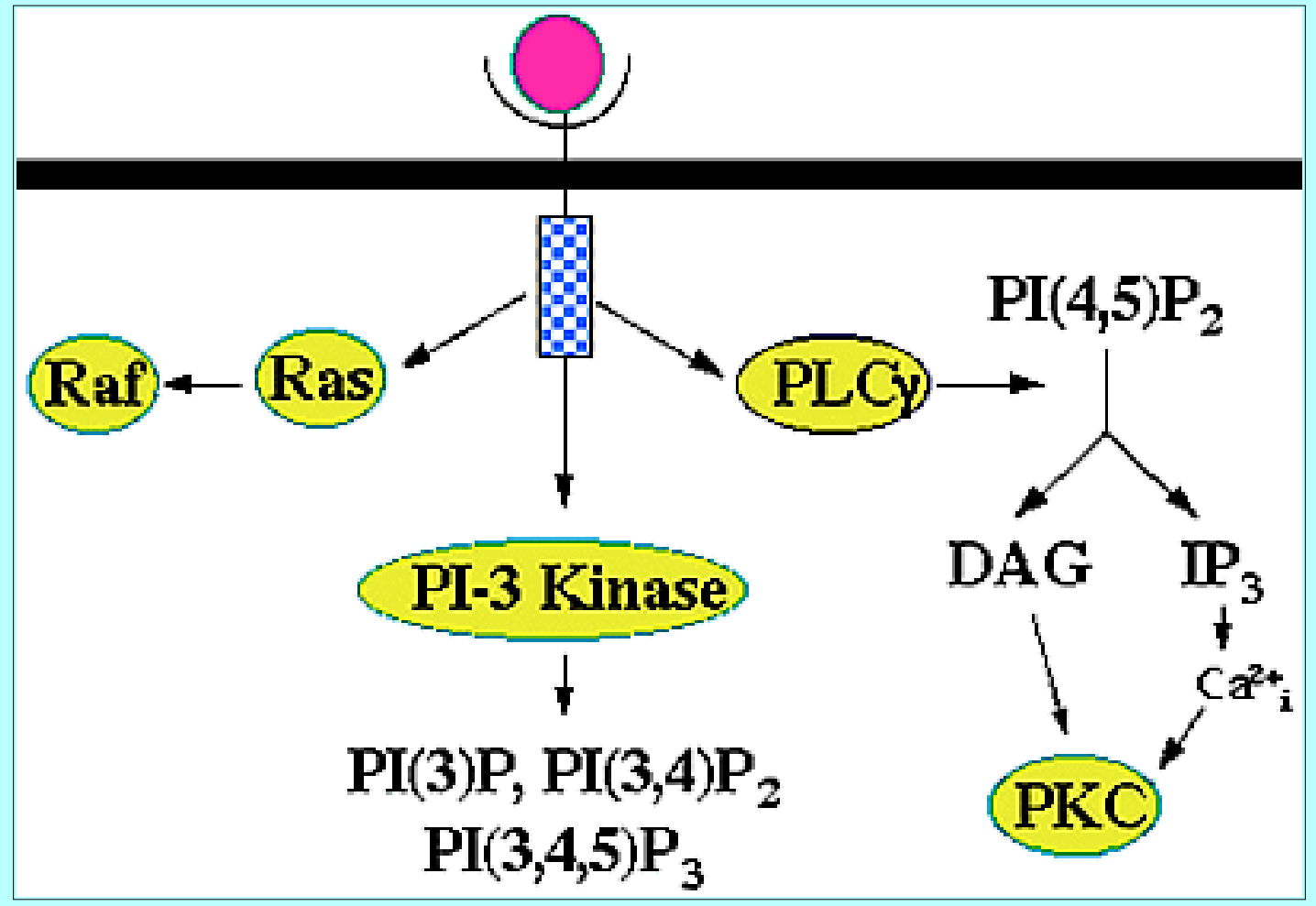

Fig. 1. Schematic showing signal transduction pathways available to cells following stimulation of a TKR. Ligand binding yields receptor autophosphorylation, thus resulting in increased TK activity. The phosphorylated substrates can then activate subsequent downstream events. DAG = dianhydrogalactitol.

Tyrosine kinase receptors activate Ras by binding the Grb2/SOS complex, which then exchanges guanosine diphosphate for guanosine triphosphate on Ras;[3,4] Ras-guanosine triphosphate translocates the serine kinase Raf to the cell membrane where it becomes phosphorylated and activated.[26,27] The activated Raf, which is the first of three in the mitogen-activated protein kinase (MAPK) module, then activates MAPK kinase (MEK) by phosphorylation of S-218 and S-222,[2,39] and MEK, which has dual-specificity kinase function, activates MAPK or extracellular signal-regulated kinase (ERK1 and ERK2) by phosphorylating both tyrosine and threonine at the consensus TEY (T-183, Y-185) sequence.[29] Extracellular signal-regulated kinase-1,2 can translocate into the nucleus and phosphorylate the transcription factor TCF/Elk1, which then binds to the serum response factor (SRF) and c-fos promoter (Fig. 2).[12,23] The full range of ERK1 and ERK2 substrates has not yet been identified and much work remains to be done in this area. Other MAPK-type pathways exist and their role in regulating the balance between proliferation and cell death may be critical. 


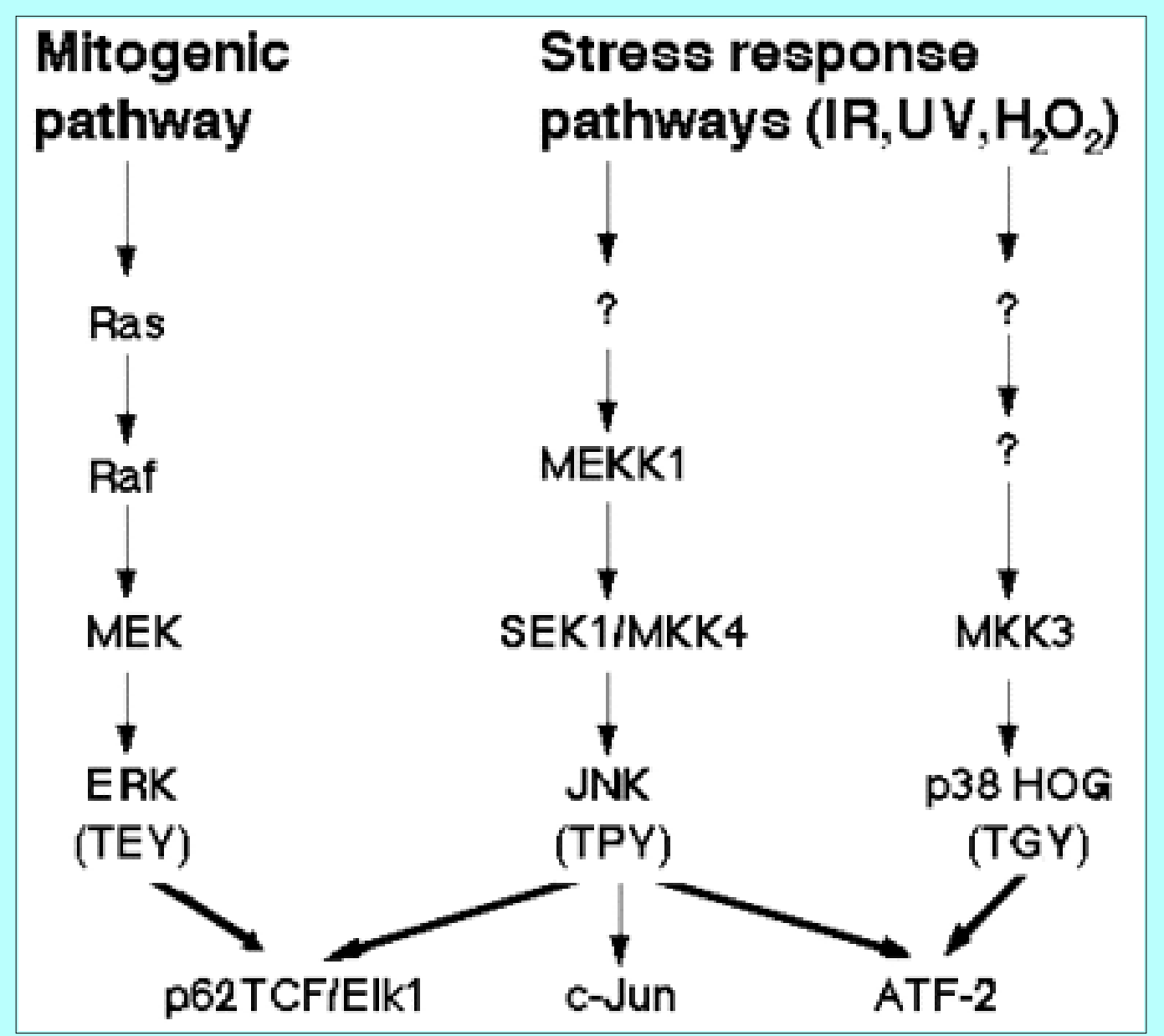

Fig. 2. Schematic showing the MAPK module kinase pathways. Three primary targets exist: ERK, JNK, and p38. These kinases translocate to the nucleus where they activate transcription factors. Both cell proliferation and apoptosis may result from activation of these pathways.

The transcription factor c-jun requires phosphorylation at S-63 and S-73 for gene transactivation.[10] This finding led to the discovery of a novel family of protein kinases (p54 and p46) similar to ERK1,2 in their requirement of tyrosine and threonine phosphorylation.[18,31] Unlike the ERKs, they are not responsive to polypeptide growth factors; rather they are activated by stressful stimuli such as ultraviolet light and inflammatory cytokines.[19] This group of proteins became known as stress-activated protein kinases (SAPK) or c-Jun $\mathrm{NH}_{2}$-terminal kinases (JNK). The JNKs are activated by a cascade of kinases analogous to the MAPK signaling module (Fig. 2) but differ slightly because they are activated by phosphorylation at a consensus TPY motif instead of TEY.[8,37] A second stress-activated pathway is based on p38-HOG, which is activated at a TGY motif.[37] The normal physiological role of SAPKs has not been investigated in gliomas. Nerve growth factor-deprived PC-12 cells undergo apoptosis and induce JNK, an effect that is reversed with transfection of a dominant negative JNK mutant.[38]

\section{CONCLUSIONS}

Growth factors and their receptors, specifically EGF, FGF, PDGF, neurotrophins, IGF, and their receptors are important in CNS development. These same factors and receptors, with the important addition of VEGF/VEGFR, seem to play a significant role in the development of CNS neoplasia. The role of these growth factors and the nuances of their expression still need further delineation before their clinical significance can be appreciated. 


\section{References}

1. Aaronson SA: Growth factors and cancer. Science 254:1146-1153, 1991

2. Alessi DR, Saito Y, Campbell DG: Identification of the sites in MAP kinase kinase-1 phosphorylated by p74raf-1. EMBO J 13:1610-1619, 1994

3. Bonfini L, Karlovich CA, Dasgupta C, et al: The Son of sevenless gene product: a putative activator of Ras. Science 255:603-606, 1992

4. Bowtell D, Fu P, Simon M, et al: Identification of murine homologues of the Drosophila son of sevenless gene: potential activators of ras. Proc Natl Acad Sci USA 89:6511-6515, 1992

5. Chao MV: Neurotrophin receptors: a window into neuronal differentiation Neuron 9:583-593, 1992

6. Chin LS, Murray SF, Zitnay KM, et al: K252a inhibits proliferation of glioma cells by blocking platelet-derived growth factor signal transduction. Clin Cancer Res 3:771-776, 1997

7. Cross M, Dexter TM: Growth factors in development, transformation, and tumorigenesis. Cell 64:270-280, 1991

8. Derijard B, Hibi M, Wu IH, et al: JNK1: a protein kinase stimulated by UV light and Ha-Ras that binds and phosphorylates the c-Jun activation domain. Cell 76:1025-1037, 1994

9. Downward J: Control of Ras activation, in Parker P, Pawson T (eds): Cell Signalling. Cancer Surveys, Vol 27. Plainview, NY: Cold Spring Harbor Laboratory Press, 1996, pp 87-100

10. Franklin CC, Sanchez V, Wagner F, et al: Phorbol ester-induced amino-terminal phosphorylation of human JUN but not JUNB regulates transcriptional activation. Proc Natl Acad Sci USA 89:7247-7251, 1992

11. Gammeltoft S, Balloti R, Kowalski A, et al: Expression of two types of receptor for insulin-like growth factors in human malignant glioma. Cancer Res 48:1233-1237, 1988

12. Gille H, Kortenjann M, Thomae O, et al: ERK phosphorylation potentiates Elk-1-mediated ternary complex formation and transactivation. EMBO J 14:951-962, 1995

13. Glick RP, Gettleman R, Patel K, et al: Insulin and insulin-like growth factor I in brain tumors: binding and in vitro effects. Neurosurgery 24:791-797, 1989

14. Hermanson M, Funa K, Hartman M, et al: Platelet-derived growth factor and its receptors in human glioma tissue: expression of messenger RNA and protein suggests the presence of autocrine and paracrine loops. Cancer Res 52:3213-3219, 1992

15. Hunter T: Protein kinases and phosphatases: the yin and yang of protein phosphorylation and signaling. Cell 80:225-236, 1995

16. Klein R, Smeyne RJ, Wurst W, et al: Targeted disruption of the trkB receptor gene results in nervous system lesions and neonatal death. Cell 75:113-122, 1993

17. Kuratsu J, Ushio Y: Antiproliferative effects of trapidil, a platelet-derived growth factor antagonist, 
on a glioma cell line in vitro. J Neurosurg 73:436-440, 1990

18. Kyriakis JM, Avruch J: pp54 microtubule-associated protein 2 kinase A novel serine/threonine protein kinase regulated by phosphorylation and stimulated by poly-L-lysine. J Biol Chem 265:17355-17363, 1990

19. Kyriakis JM, Avruch J: Sounding the alarm: protein kinase cascades activated by stress and inflammation. J Biol Chem 271:24313-24316, 1996

20. Lai C, Lemke G: An extended family of protein tyrosine kinase genes differentially expressed in the vertebrate nervous system. Neuron 6:691-704, 1991

21. Leon SP, Zhu J, Black PM: Genetic aberrations in human brain tumors. Neurosurgery 34:708-722, 1994

22. Malden LT, Novak U, Kaye AH, et al: Selective amplification of the cytoplasmic domain of the epidermal growth factor receptor gene in glioblastoma multiforme. Cancer Res 48:2711-2714, 1988

23. Marais R, Wynne J, Treisman R: The SRF accessory protein Elk-1 contains a growth factor-regulated transcriptional activation domain. Cell 73:381-393, 1993

24. Marchionni MA, Goodearl AD, Chen MS, et al: Glial growth factors are alternatively spliced erb-2 ligands expressed in the nervous system. Nature 362:312-318, 1993

25. Maxwell M, Naber SP, Wolfe HJ, et al: Coexpression of platelet-derived growth factor (PDGF) and PDGF-receptor genes by primary human astrocytomas may contribute to their development and maintenance. J Clin Invest 86:131-140, 1990

26. Morrison DK, Heidecker G, Rapp UR, et al: Identification of the major phosphorylation sites of the Raf-1 kinase. J Biol Chem 268:17309-17316, 1993

27. Morrison DK, Kaplan DR, Escobedo JA, et al: Direct activation of the serine/threonine kinase activity of Raf-1 through tyrosine phosphorylation by the PDGF beta-receptor. Cell 58:649-657, 1989

28. Morrison RS, Yamaguchi F, Bruner JM, et al: Fibroblast growth factor receptor gene expression and immunoreactivity are elevated in human glioblastoma multiforme. Cancer Res 54:2794-2799, 1994

29. Payne DM, Rossomando AJ, Martino P, et al: Identification of the regulatory phosphorylation sites in pp42/mitogen-activated protein kinase (MAP kinase). EMBO J 10:885-892, 1991

30. Peters K, Ornitz D, Werner S, et al: Unique expression pattern of the FGF receptor 3 gene during mouse organogenesis. Dev Biol 155:423-430, 1993

31. Pulverer BJ, Kyriakis JM, Avruch J, et al: Phosphorylation of c-jun by MAP kinases. Nature 353:670-674, 1991

32. Schlessinger J, Ullrich A: Growth factor signaling by receptor tyrosine kinases. Neuron 9:383-391, 1992

33. Ullrich A, Schlessinger J: Signal transduction by receptors with tyrosine kinase activity. Cell 61:203-212, 1990 
34. Vescovi AL, Reynolds BA, Fraser DD, et al: bFGF regulates the proliferative fate of unipotent (neuronal) and bipotent (neuronal/astroglial) EGF-generated CNS progenitor cells. Neuron 11:951-966, 1993

35. Weiner HL: The role of growth factor receptors in central nervous system development and neoplasia. Neurosurgery 37:179-194, 1995

36. Wong AJ, Bigner SH, Bigner DD, et al: Increased expression of the epidermal growth factor receptor gene in malignant gliomas is invariably associated with gene amplification. Proc Natl Acad Sci USA 84:6899-6903, 1987

37. Woodgett JR, Avruch J, Kyriakis JM: The stress activated protein kinase pathway, in Parker P, Pawson T, (eds): Cell signalling. Cancer Surveys, Vol 27. Plainview, NY: Cold Spring Harbor Laboratory Press, 1996, pp 127-138

38. Xia Z, Dickens M, Raingeaud J, et al: Opposing effects of ERK and JNK-p38 MAP kinases on apoptosis. Science 270:1326-1331, 1995

39. Zheng CF, Guan KL: Activation of MEK family kinases requires phosphorylation of two conserved Ser/Thr residues. EMBO J 13:1123-1131, 1994

Manuscript received July 15, 1997.

Accepted in final form August 20, 1997.

Address reprint requests to: Lawrence S. Chin, M.D., Department of Neurosurgery, University of Maryland, 22 South Greene Street, Baltimore, Maryland 21201. 